\title{
Challenges, Strategies and Opportunities for Measuring Carbon Nanotubes within a Polymer Composite by X-ray Photoelectron Spectroscopy
}

Version 1.0

J. M. Gorham

J. W. Woodcock

K. C. Scott 


\section{Challenges, Strategies and Opportunities for Measuring Carbon Nanotubes within a Polymer Composite by X-ray Photoelectron Spectroscopy}

Version 1.0

J. M. Gorham

K. C. Scott

Material Measurement Science Division

Material Measurement Laboratory

J. W. Woodcock Material Science and Engineering Division Material Measurement Laboratory

This publication is available free of charge from: http://dx.doi.org/10.6028/NIST.SP.1200-10

March 2015

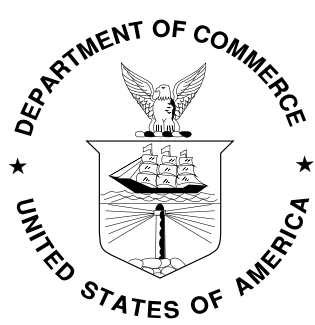

U.S. Department of Commerce Penny Pritzker, Secretary

National Institute of Standards and Technology Willie May, Acting Under Secretary of Commerce for Standards and Technology and Acting Director 
Certain commercial entities, equipment or materials may be identified in this document in order to describe an experimental procedure or concept adequately. Such identification is not intended to imply recommendation or endorsement by the National Institute of Standards and Technology, nor is it intended to imply that the entities, materials or equipment are necessarily the best available for the purpose. 


\section{FOREWORD}

This NIST Special Publication (SP) is one in a series of NIST SPs that address research needs articulated in the National Nanotechnology Initiative (NNI) Environmental, Health, and Safety Research Strategy (EHS) published in 2011 [1]. This Strategy identified a Nanomaterial Measurement Infrastructure (NMI) as essential for science-based risk assessment and risk management of nanotechnology-enabled products as pertaining to human health, exposure, and the environment. NIST was identified as the lead federal agency in the NMI core research area of the Strategy. This research area includes development of measurement tools for the detection and characterization of engineered nanomaterials in nanotechnology-enabled products. Carbon nanotubes (CNTs) in commercial products, the focus for this special publication, were one of the nanomaterials identified in the Nano-EHS research strategy.

This SP presents some challenges and strategies for the characterization of CNT:polymer composite systems by X-ray Photoelectron Spectroscopy (XPS). This SP outlines surface preparations and identifies hurdles associated with the measurement of polymer composites, and provides some solutions towards gaining qualitative to semi-quantitative XPS, depending on the scenario. The authors do not claim that (A) this is an exhaustive list of all challenges associated with XPS measurements of composites or (B) there are no other acceptable solutions to some of the noted challenges. Updates to this protocol may be released in the future. Visit http://nist.gov/mml/np-measurement-protocols.cfm to check for revisions of this protocol, or new protocols in the series. We also encourage users to report citations to published work in which this protocol has been applied. 


\section{Introduction}

Multi-walled carbon nanotubes (MWCNTs) are widely studied nanomaterials due to the unique properties they possess, including mechanical strength and electrical conductivity. As a result, MWCNTs have been incorporated in new and existing commercial products to impart and/or enhance a desired physico-chemical property. One class of materials that takes advantage of these enhanced properties are polymer:MWCNT containing composites which are used in protective apparels, automotive applications and sporting equipment. Concerns about human exposure to MWCNTs have given rise to a need for characterization of MWCNTs in products. However, detection of MWCNTs within commercial products can be quite challenging due to sample unknowns, including MWCNT concentration, identification of the polymer, and the identity of other additives deemed proprietary by the manufacturer. Researchers at NIST have worked to characterize composites using an MWCNT:epoxy based composite in effort to develop characterization and detection strategies for MWCNTs in commercial products.

\section{Principles and Scope}

One of the most commonly employed analytical tools for characterization of the elemental and chemical properties at or near a surface (within $10 \mathrm{~nm}$ ) is X-ray photoelectron spectroscopy (XPS). The technique has been previously explained in detail $[2,3]$ and information obtained from this technique may include the detection of surface elements, the identification of their chemical functionality and oxidation state, and semi-quantitative to quantitative characterization of their surface concentrations. XPS has been employed in the characterization of a multitude of nanomaterials, including in MWCNTs alone and within composites [4-6]. The purpose of this special publication is to identify challenges and, when possible, solutions associated with preparation and XPS characterization of MWCNT:polymer composites. Many of the approaches mentioned here can be applied to other nanocomposites. It is also important to note that this may not be an exhaustive list of all problems and solutions associated with this technique for MWCNT:epoxy composites.

\section{Materials and equipment}

\subsection{XPS}

3.1.1 Technical Description: XPS is a highly surface sensitive technique which acquires information on the elemental and chemical composition of the top $10 \mathrm{~nm}$ of a surface, depending on the energies of the X-ray and the photoelectron [2]. From a twodimensional standpoint, an XP spectrum provides the average elemental surface concentration over an area with a spot size diameter of (10's to 100's) of micrometers. Its depth of analysis, however, makes it ideal for characterization of nanoscale surface structures and particles. Due to its utility in nanocharacterization and other disciplines, it is no surprise that the number of publications on XPS has been steadily rising over the past two decades $[7,8]$.

XPS employs a well-defined source of X-rays of energy $h v$ directed towards a surface. As a result, a photon ejects a core level electron from an element $(n)$ in the sample which is analyzed according to its kinetic energy $\left(\mathrm{KE}_{n}\right)$ and counted. In order to identify the element in question, a binding energy $\left(\mathrm{BE}_{n}\right)$ can be calculated for the element using the following, extremely simplified, equation:

$$
\mathrm{BE}_{n}=\mathrm{h} v-\mathrm{KE}_{n}+\phi
$$


The work function, $\phi$, is an additional factor associated with the energy needed to eject a photoelectron from the Fermi level and into the spectrometer. The BE of any elemental transition is specific to the orbital from which the photoelectron originated and can be impacted by its local chemical environment and oxidation state. Photoelectrons can be analyzed for all elements, except for hydrogen and helium. Further explanation of the photoemission process, work functions and characterization using XPS can be found in other texts [2].

3.1.2 Examples of spectra acquired for this SP will either be from the literature or have been acquired on an Axis Ultra DLD spectrophotometer from Kratos Analytical (Chestnut Ridge, NY). New spectra were acquired with monochromatic Al Ka X-rays with photoelectrons collected along the surface normal and filtered using a hemispherical analyzer.

\subsection{Starting materials/samples}

The creation of MWCNT composites using a two part epoxy has been discussed in the literature with synthetic techniques fully explained [4-6, 9]. However, the MWCNTs employed in new spectra found in this SP were MWCNTs from Arkema (King of Prussia, PA). The epoxy matrix was composed of diglycidal ether of bisphenol A (DGEBA) and a Jeffamine curing agent. Samples were allowed to cure at $80^{\circ} \mathrm{C}$ in a silicone mold. Control samples were generated for the MWCNTs and the 2 part epoxy, separately, using the same materials as previously mentioned.

\subsection{Sample Preparation equipment}

3.3.1 Surface modifications -During the curing of MWCNT:epoxy composites, a polymer rich film may form at the surface which is inconsistent with the bulk composition. For a surface sensitive technique such as XPS, this thin film can block the acquisition of a representative measurement of the majority of the sample. There are a few options for removing this surface layer.

3.3.1.1 Rotary microtome - This technique employs a fine diamond blade to physically remove the topmost layer of a sample. The microtome excels at creating a highly smooth surface that is not chemically altered. However, the prepared surface area is typically on the order of 10's to 100's of square micrometers. While this is measurable by XPS, the small analysis area may provide insufficient photoelectrons to resolve low elemental concentrations at the surface.

3.3.1.2 Ion milling - This technique employs high energy ion beam using an inert gas (e.g. argon) to remove the top layers of a sample. A key benefit to this process is that it is done in-situ, preventing surface contamination. Additionally, the sample can be cleaned again if necessary quickly and efficiently. Unfortunately, ion milling can damage surface chemical functionalities, especially in polymers.

3.3.1.3 End-milling - This technique employs a machining tool, such as a drill, to remove the top layer of a composite. One benefit is the exposure of a large cleaned area of composite in a relatively short time. Additionally, there is minimal chance for chemical modifications. Some disadvantages may include the rough morphology and topographic features that the machining tools may leave behind. 
3.3.2 Preliminary pumpdown chambers $\left(\mathrm{P}_{\text {base }}<10^{-5} \mathrm{~Pa}\left(<10^{-7}\right.\right.$ torr $\left.)\right)$ - Electron spectroscopy techniques require UHV pressures $\left(<10^{-6} \mathrm{~Pa}\left(<10^{-8}\right.\right.$ torr $\left.)\right)$. Extended pumpdown times in a preliminary chamber may be required to achieve UHV conditions due to absorbed water, residual precursors and trapped gas pockets at the surface of a sample. Typically, these chambers are attached to the XPS chamber and are equipped with a combination of a rough pump and a turbomolecular pump. Alternatively, a separate chamber evacuated with a rough pump can also be employed to do some preliminary outgassing.

3.3.3 Sample bars - Typically, samples are loaded onto a sample bar or stub and fixed to the surface. For MWCNT:epoxy composites, holding the sample down with conducting fastener tabs is preferred in order to maximize the routes of charge dissipation; either through the sample or across the sample surface.

\section{Challenges, considerations, solutions and opportunities}

\subsection{Sample Preparation Challenges}

\subsubsection{Sample geometry: lateral size}

The minimum area of analysis corresponds to the optimized spot size, typically on the order of (10's to 100's) of square micrometers, as dictated by the analyzer's optics, which is an important factor to understand when analyzing small specimens. However, the relatively larger area of analysis corresponds to improved signal, a higher signal to noise ratio and shorter time to acquire the data. This is important for poor photoemitters like carbon. Additionally, the sample should be substantially larger than the area of analysis to avoid signal from the sample holder. Considerations should be made for the following:

Consideration 1: What is the suspected MWCNT concentration? If the sample was prepared in-house, then this should be already known. For a commercial product, the manufacturer should be consulted in attempts to gain an estimate. The smaller the percent MWCNT added, the larger the area of analysis to obtain sufficient signal for a given amount of time. XPS is typically useful for concentrations $>0.1$ atomic\%. Another way of enhancing the signal can be to boost the flux of X-rays incident upon the surface.

Consideration 2: How much time is available for data acquisition? Smaller areas of analysis will take longer to obtain quality spectra. If one is acquiring spectra for samples of a lower MWCNT concentration, it is necessary to schedule more time to obtain quality spectra from smaller samples.

\subsubsection{Sample geometry: settling concerns}

One of the steps for making MWCNT: Epoxy composites involves placing the mixture into a mold and allowing the composite to cure. It has been observed with other nanomaterial:epoxy composites that the final product has a 'nano-free' thin film (thickness > $10 \mathrm{~nm}$ ) composed largely of epoxy and not representative of the bulk $[4,6$, 10]. As a result, interrogating the unmodified nanocomposite surface/interface provides limited benefits without additional sample preparation.

Solution: Remove surface layer - In order to study the representative bulk nanocomposite, the epoxy rich layer has to be removed. Tools to accomplish this are discussed in 3.3.1. 


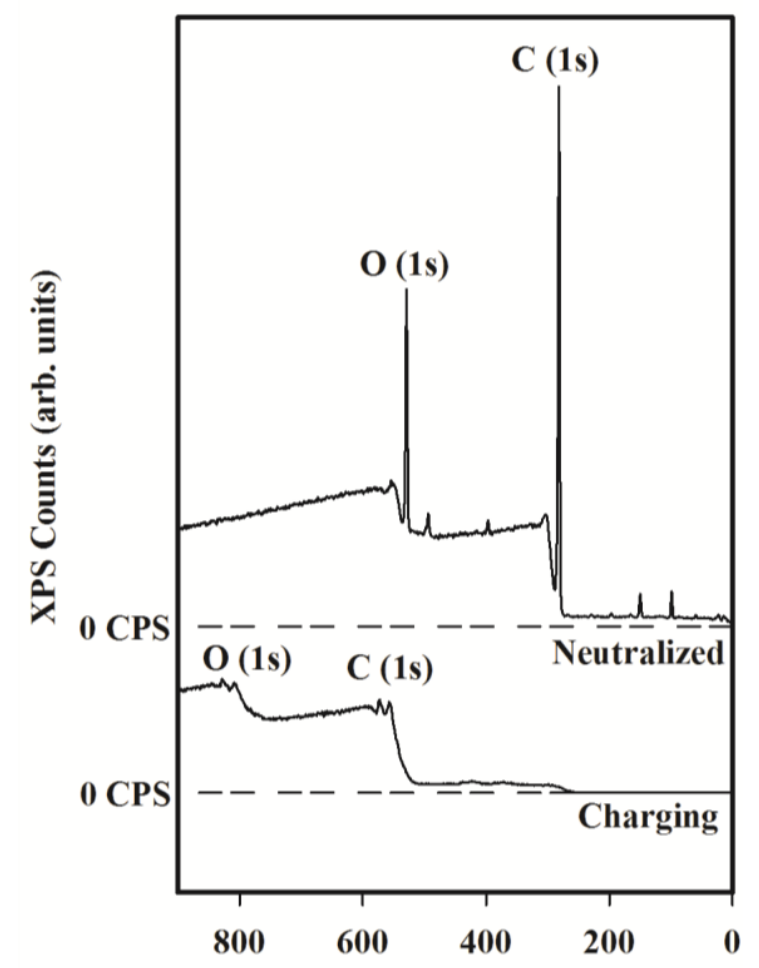

Binding Energy (eV)

Figure 1: XPS spectra of DGEBA epoxy before (charging) and after (neutralized) exposure to a low energy electron source. Charge neutralized spectra are characterized by sharp peaks representative of photoelectrons from elemental orbitals. Charging spectra is characterized by only the strongest photoelectron peaks (carbon and oxygen) appearing at locations shifted to higher binding energy.

min to 15 min under a rough vacuum.

Solution 3 - Pump down in a separated preliminary chamber: The safest way to minimize outgassing in an XPS analysis chamber is to pump the sample down in a preliminary pump down chamber (see 3.3.2). Most XPS systems have these as a point of entrance separated by a UHV compatible valve. Depending on the samples, this could be quite time intensive taking several hours to several days.

\subsection{Sample Characterization Challenges}

\subsubsection{Charging due to low conductivity of the polymeric material}

While not universally true, many polymeric materials can be electrically insulating, including epoxy based materials. In well dispersed composites, when the MWCNT mass fraction is sufficiently low, the sample may charge during data acquisition. In XPS, charging occurs when a core level vacancy is created and is not replaced due to the insulating properties of the material. This causes a positive bias build up which impacts photoemission and can preclude semi- 
quantitative elemental and chemical analysis. An example of charging can be observed in Figure 1 (Bottom).

Solution-Charge neutralizers: Charge neutralizers are low energy electron flood sources (typically $<20 \mathrm{eV}$ [2]) designed to replace lost photoelectrons. Figure 1 (top) demonstrates the impact of applying a charge neutralizer to an insulating sample. This is also reflected in Figure $2 \mathrm{~A}$ for higher resolution scans. When using a charge neutralizer, one must be certain to correct for over adjustment which results in peaks shifted to lower binding energies. This can be accomplished by adjusting the binding energy scale with a known transition. In most charging cases, neutralization is sufficient to allow for elemental quantification. Chemical analysis and assignment of different functionalities, however, will depend on the sample.

\subsubsection{Differential charging: This} phenomenon occurs when a sample is made of two materials that have significantly different electrical properties. In the example of polymer composites, MWCNTs are typically conductive while the polymer is more insulating.
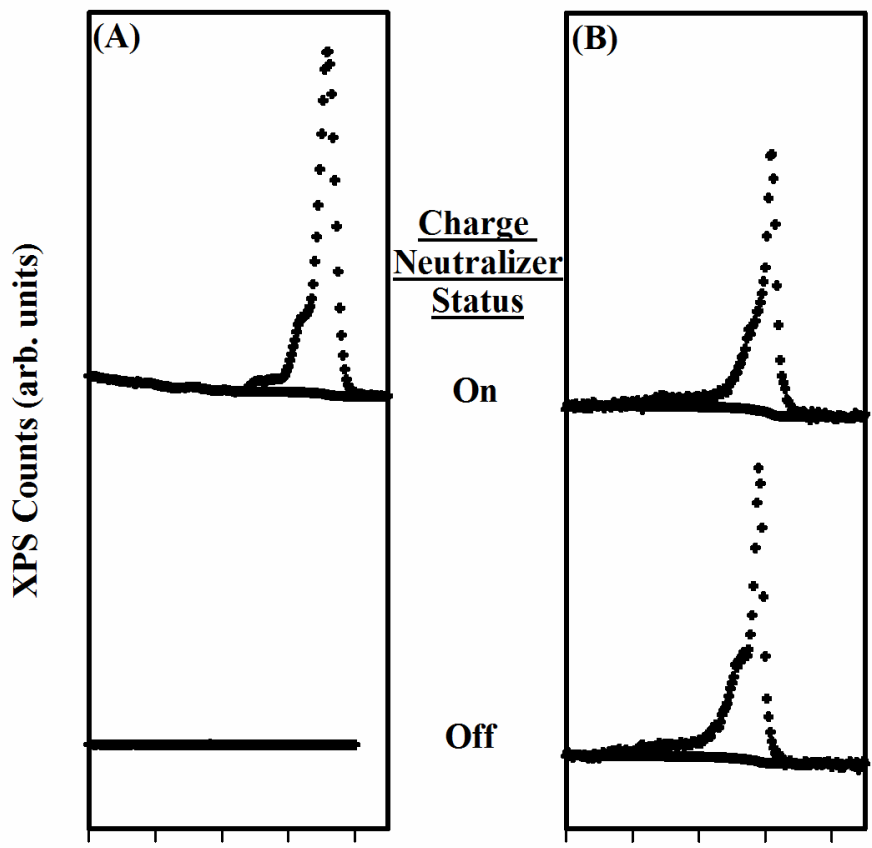

296292288284280

Binding Energy (eV)

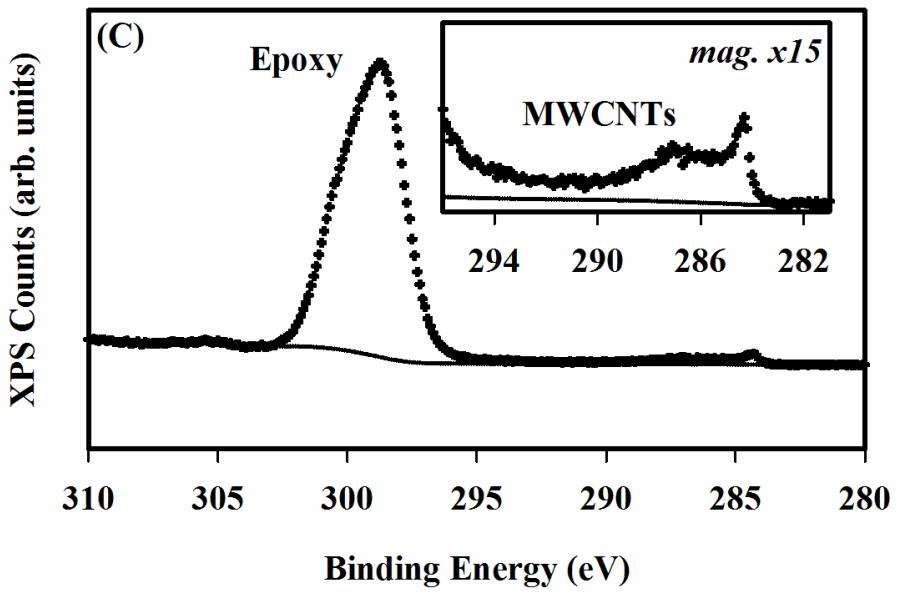

Figure 2: XP spectra looking at the difference between the charge neutralizer on (top/neutralized) or off (bottom/charging) (A) for DGEBA epoxy and (B) for MWCNTs. (C) Demonstrates differential charging for $1 \%$ MWCNT:Epoxy composite. (Inset) Zoom in of the MWCNT region magnified $15 \mathrm{x}$

This can result in a splitting of the $\mathrm{C}$ (1s) signals (see Figure 2C). Charge neutralizers are of limited use in the case of differential charging since they overcompensate insulators (Figure 2A) and have minimal impact on conductors (Figure 2B). While semiquantitative elemental analysis is still possible, chemical assignment of different functionalities may be untenable. 


\begin{tabular}{|c|c|c|c|}
\hline $\begin{array}{c}\text { Sample } \\
\text { materials }\end{array}$ & $\begin{array}{l}\text { Graphene, Single walled } \\
\text { CNTs, MWCNTs, } \\
\text { Functionalized MWCNTs }\end{array}$ & $\begin{array}{l}\frac{\text { Bulk Scale }}{\text { Graphite and/or }} \\
\text { HOPG }\end{array}$ & $\begin{array}{l}\text { Polyvinyl alcohol, poly(ethylene), } \\
\text { poly(styrene), polyphenylene oxide, } \\
\text { DGEBA epoxy, alkanethiols, PVC }\end{array}$ \\
\hline BE & (CH-CC) C (1s) peak max & $\begin{array}{l}\text { (CH-CC) C (1s) } \\
\text { peak Max }\end{array}$ & (CH-CC) C (1s) Peak Max \\
\hline \multicolumn{4}{|l|}{283.9} \\
\hline 284.0 & [11] [12] & & [13] \\
\hline \multicolumn{4}{|l|}{284.1} \\
\hline 284.2 & [12] & [12] & \\
\hline 284.3 & & [14] & \\
\hline 284.4 & [12] & & \\
\hline 284.5 & [12] [6] [5] & [15] & \\
\hline 284.6 & [16] [17] [3] & & [18] [19] [20] [21] \\
\hline 284.7 & & [22] [23] & \\
\hline 284.8 & & & [10] [24] \\
\hline \multicolumn{4}{|l|}{284.9} \\
\hline 285.0 & [12] & & [25] [26] [27] \\
\hline 285.1 & [12] & & {$[28]$} \\
\hline 285.2 & & & \\
\hline
\end{tabular}

Table 1: References for nanoscale graphitic carbon (Left), bulk scale graphitic carbon (Center) and a variety of hydrocarbon peaks (Right) are presented. On average, graphitic carbon references place the $\mathrm{C}$ (1s) peak max at $(284.5+/-0.3) \mathrm{eV}$ while hydrocarbon was centered at $(284.7+/-0.3) \mathrm{eV}$, based on the average +/- 1 standard deviation of the above examples. Highlighted references are those in which energy was referenced to the $\mathrm{C}(1 \mathrm{~s})$ peak maximum due to charging and no other viable option.

\subsubsection{Peak overlaps between graphitic and hydrocarbon components}

When carbonaceous samples possess uniform electrical properties, fitting the $\mathrm{C}(1 \mathrm{~s})$ region with a background and determining the functionalities present at the surface is very common. Typically this is achieved by using various peaks at different binding energies (e.g. combinations of Gaussian and/or Lorentzian curves). However, a major problem is that the hydrocarbon in polymers and the graphitic layers of the carbon nanotubes have comparable peak locations (Table 1) with an average peak separation of $\approx 0.2 \mathrm{eV}$. Furthermore, the oxide peaks in the epoxy overlap with the with the MWCNT tail (See Figure 2(B)). In most cases, the authors do not recommend trying to separate hydrocarbon features from graphitic functionalities by peak fitting. However, the following steps should be considered if one is set to attempt it.

Consideration 1-Calibrate analyzer: While considered good practice generally, it is important to calibrate the energy scale in this scenario. It is advised to employ accepted methods and standards from the literature. Some examples of where information may be found are in ISO 10810 and ISO 15472.

Consideration 2 - Identify an independent elemental marker: This element (e.g. noble metals) should be employed for binding energy scale adjusted for small sample to sample variations in conductivity and work-function. 
Consideration 3 - Run controls for reference peak positions: As demonstrated in Table 1, literature references are typically for just one species and are based on the instrument from which they were acquired. Substrate effects [12, 29], analyzer settings, small variances in the conductivity, fitting vs. raw spectra and calibration settings are just a few of the sources of slight energy shifts that will impact assignment of peak positions for MWCNTs and hydrocarbon. As observed in Table 1, two different references may provide peak locations anywhere from $0.1 \mathrm{eV}$ to $1.1 \mathrm{eV}$ apart.

\subsection{Chemical information available from XPS characterization of MWCNT composites despite differential charging and spectral overlap challenges}

While charging in XPS can be detrimental towards effectively characterizing a sample set in a quantitative fashion, there are opportunities for employing it to obtain some information on the chemical nature of a given sample in a qualitative or semi-quantitative fashion. One example of this can be observed in a previous aging study where the surface accumulation of MWCNTs was noted using XPS methodologies [6]. As a result of allowing the differential charging to occur, the epoxy surface component was allowed to shift to higher binding energies while the conductive MWCNTs could be observed, unskewed. An example of this methodology can be observed in Figure 2C and was also employed in other publications [4].

A second example of employing differential charging to characterize the surface contributions from MWCNTs by XPS was in a recent study by Goodwin et al. [5] where CNT:Polyvinyl alcohol (PVA) composites were employed with varying CNT mass fraction added. XPS revealed only a small degree of differential charging resulting in a small shift in the PVA component of the specimens $(\approx 1 \mathrm{eV})$ from the MWCNT component and no observable skewing of the spectra. By characterizing pure PVA, varying the MWCNT composition of composites, and $100 \%$ MWCNT controls, the composites could be characterized with trends observable in a semi-quantitative fashion.

It is important to note that in both of these cases, large numbers of samples and good controls were necessary to successfully extract usable qualitative and semi-quantitative information from these MWCNT composites by XPS.

\section{Acknowledgements}

We would like to thank Debra Kaiser for her direction and vision in the characterization of nanomaterials in consumer products, from which the development of this protocol derived. 
6 References

1. National Nanotechnology Initiative: Environmental, Health and Safety Research Strategy. 2012, Presidents Council of Advisors on Science and Technology: National Science and Technology Council Committee on Technology: Washington, DC.

2. Vickerman, J.C. and I.S. Gilmore, Surface analysis : the principal techniques. 2nd ed. 2009, Chichester, U.K.: Wiley. xix, 666 p.

3. Wepasnick, K.A., et al., Chemical and structural characterization of carbon nanotube surfaces. Analytical and Bioanalytical Chemistry, 2010. 396(3): p. 1003-1014.

4. Ging, J., et al., Development of a conceptual framework for evaluation of nanomaterials release from nanocomposites: Environmental and toxicological implications. Science of the Total Environment, 2014. 473: p. 9-19.

5. Goodwin Jr, D.G., et al., Interactions of Microorganisms with Polymer Nanocomposites Containing Different Loadings of Oxidized Single and Multi-wall Carbon Nanotubes. submitted to Environmental Science and Technology, 2015.

6. Petersen, E.J., et al., Methods to assess the impact of UV irradiation on the surface chemistry and structure of multiwall carbon nanotube epoxy nanocomposites. Carbon, 2014. 69: p. 194205.

7. Powell, C.J., Growth and trends in Auger-electron spectroscopy and x-ray photoelectron spectroscopy for surface analysis. Journal of Vacuum Science \& Technology A: Vacuum, Surfaces, and Films, 2003. 21(5): p. S42.

8. Powell, C.J. and A. Jablonski, Surface Sensitivity of Auger-Electron Spectroscopy and X-ray Photoelectron Spectroscopy. Journal of Surface Analysis, 2011. 17(3): p. 170-176.

9. Davis, C.S., J.W. Woodcock, and J.W. Gilman, Preparation of Nanoscale Multiwalled Carbon Nanotube Dispersions in a Polyetheramine Epoxy for Eco-Toxicological Assessment. NIST Special Publication, submitted 2014.

10. Gorham, J.M., et al., Photo-induced surface transformations of silica nanocomposites. Surface and Interface Analysis, 2012. 44(13): p. 1572-1581.

11. Ma, P.-C., et al., Dispersion, interfacial interaction and re-agglomeration of functionalized carbon nanotubes in epoxy composites. Carbon, 2010. 48(6): p. 1824-1834.

12. Preobrajenski, A., et al., Controlling graphene corrugation on lattice-mismatched substrates. Physical Review B, 2008. 78(7).

13. Way, W.K., Polystyrene by XPS. Surface Science Spectra, 1993. 2(1): p. 67.

14. Vasquez, R.P., Highly Oriented Pyrolytic Graphite by XPS. Surface Science Spectra, 1992. 1(2): p. 238.

15. Crist, B.V., Argon Implanted into Graphite, by XPS. Surface Science Spectra, 1992. 1(4): p. 376.

16. Adhikari, A.R., et al., Stability of ion implanted single-walled carbon nanotubes: Thermogravimetric and Raman analysis. Journal of Applied Physics, 2006. 100(6): p. 064315.

17. Larrude, D.G., et al., Characterization of phosphorus-doped multiwalled carbon nanotubes. Journal of Applied Physics, 2012. 111(6): p. 064315.

18. Gorham, J., B. Smith, and D.H. Fairbrother, Modification of alkanethiolate self-assembled monolayers by atomic hydrogen: Influence of alkyl chain length. Journal of Physical Chemistry C, 2007. 111(1): p. 374-382.

19. Gorham, J., et al., Surface reactions of molecular and atomic oxygen with carbon phosphide films. Journal of Physical Chemistry B, 2005. 109(43): p. 20379-20386.

20. Burrell, M.C., Polyphenylene Oxide (PPO) Spin Cast Films by XPS. Surface Science Spectra, 1999. 6(1): p. 9.

21. Burrell, M.C., Polystyrene Spin Cast Films by XPS. Surface Science Spectra, 1999. 6(1): p. 27. 
22. Schmieg, S.J., Highly Oriented Pyrolytic Graphite by XPS. Surface Science Spectra, 1992. 1(4): p. 333.

23. Xie, Y., Highly Oriented Pyrolytic Graphite by Core Level and Valence Band XPS. Surface Science Spectra, 1992. 1(3): p. 253.

24. Persson, H.H.J., W.R. Caseri, and U.W. Suter, Versatile Method for Chemical Reactions with SelfAssembled Monolayers of Alkanethiols on Gold. Langmuir, 2001. 17(12): p. 3643-3650.

25. Louette, P., F. Bodino, and J.-J. Pireaux, Poly(ethylene) (PE) XPS Reference Core Level and Energy Loss Spectra. Surface Science Spectra, 2005. 12(1): p. 49.

26. Louette, P., F. Bodino, and J.-J. Pireaux, Poly(vinyl alcohol) (PVA) XPS Reference Core Level and Energy Loss Spectra. Surface Science Spectra, 2005. 12(1): p. 106.

27. Yang, Y.W. and L.J. Fan, High-Resolution XPS Study of Decanethiol on Au(111): Single Sulfur-Gold Bonding Interaction. Langmuir, 2002. 18(4): p. 1157-1164.

28. Lannon, J.M., Analysis of a Filled Poly(vinyl chloride) Polymer by XPS. Surface Science Spectra, 1999. 6(2): p. 131.

29. Dahal, A., et al., Charge doping of graphene in metaVgraphene/dielectric sandwich structures evaluated by C-1s core level photoemission spectroscopy. APL Materials, 2013. 1(4): p. 042107. 